\title{
DIFFICULTIES IN THE DIAGNOSIS AND MANAGEMENT OF HYPONATREMIA
}

\author{
MIHAELA MOCAN, LIA MANUELA TERHEŞ, SORIN NICU BLAGA
}

\author{
Department of Internal Medicine, $1^{\text {st }}$ Medical Clinic, Iuliu Hatieganu University \\ of Medicine and Pharmacy, Cluj-Napoca, Romania
}

\begin{abstract}
Hyponatremia is the most common electrolyte imbalance encountered in clinical practice. Beside the difficulty of the etiological diagnosis, the general lack of awareness regarding the negative impact of hyponatremia on the quality of life and the lack of targeted treatments until recent years may be responsible for the poor management of the disorder.

Therefore we consider of utmost importance to improve the knowledge regarding the diagnosis and management of hyponatremia, by underlining the difficulties of the etiological diagnosis and its management.

In this respect, a short and updated literature review, with a critical appraisal of the latest guidelines will provide detailed information on how to systematically evaluate and treat the hyponatremic patients.
\end{abstract}

Keywords: hyponatremia, inappropriate ADH syndrome, vasopressin antagonists, water-electrolyte imbalance

\section{Introduction}

Hyponatremia (serum $\mathrm{Na}$ levels of $<135 \mathrm{mEq} / \mathrm{l}$ ), is the most common electrolyte imbalance encountered in clinical practice, affecting up to $15-28 \%$ of hospitalized patients [1]. Its incidence varies between hospitals and departments, with a frequency of $36 \%$ in internal medicine department, very similar to that found in surgical or intensive care departments, as showed by Hoorn et al. [2]. Both moderate and especially severe hyponatremia $(\mathrm{Na}<125$ $\mathrm{mEq} / \mathrm{l}$ ) found in newly admitted hospital patients is linked with a significantly elevated in-hospital mortality of $28 \%$ compared to $9 \%$ in-hospital mortality in normonatremic, matched controls [3]. Mortality in fact increases when serum $\mathrm{Na}$ levels are below $135 \mathrm{mEq} / \mathrm{l}$. Patients with hyponatremia also have varied clinical presentations that include differing symptomatology, underlying etiology and fluid volume status.

Despite frequently observed in hospitalized patients, the diagnosis and management of hyponatremia is neither easy nor optimal [4]. This may be attributable to

Manuscript received: 10.12.2015

Accepted: 17.01.2016

Address for correspondence: mihaela.mocan@gmail.com the diversity of underlying disease states associated with the condition and, until the last few years, a lack of targeted treatments.

Thus, we consider of utmost importance to improve the knowledge regarding the diagnosis and management of hyponatremia. In this respect, a short and updated literature review will provide detailed information on the diagnosis and management of hyponatremic patients.

\section{Clinical presentation}

Neurological symptoms of hyponatremia (gait disturbances, cognitive dysfunction and dizziness) may lead to falls and subsequent injuries requiring medical care. The symptoms are subtle, difficult to diagnose and rarely identified as determined by hyponatremia, especially in older patients. Even though cognitive and/ or geriatric functional tests regularly reveal a significant impairment in patients with hyponatremia as compared to normonatremic ones, hyponatremia is often overseen or not given full attention [3]. Furthermore, if hyponatremia is diagnosed, it is regularly classified to be asymptomatic and the underlying reasons often remain obscure [4]. Thus, the etiology of hyponatremia needs to be systematically 
determined in order to apply rapid and correct therapeutic sanctions.

Two factors influence the severity of the clinical picture:

- biochemical severity based on concentration of sodium in serum:

- mild: $130-135 \mathrm{mEq} / 1$

- moderate: $125-129 \mathrm{mEq} / 1$

- severe: $<125 \mathrm{mEq} / 1$

- $\quad$ speed of development

- acute (usually $<48 \mathrm{~h}$ )

- chronic [5].

Clearly, the symptoms of hyponatremia depend on the time lapsed since the start of hyponatremia. Hyponatremia with rapid onset $(<48 \mathrm{~h})$ is associated with severe symptoms caused by cerebral edema and high intracranial pressure: epileptic convulsions, pronounced somnolence or coma, vomiting and compromised respiratory regulation. These patients sometimes die of brain herniation.

In chronic hyponatremia, brain cells extrude organic solutes from their cytoplasm, allowing intracellular osmolality to equal plasma osmolality without a large increase in cell water. Therefore, patients with chronic ( $>48$ hours) hyponatremia have more modest symptoms and almost never die of brain herniation. Symptoms like headache, modest nausea in general reflect a more moderate severity associated with insidious onset [5].

Thus, a brief anamnesis (regarding the medical history, physical activity, drugs) and physical examination (hydration status, cardiovascular status, neurological exam) should be performed (Table I) in a patients with acute hyponatremia. Usually, the clinical picture is correlated with hyponatremia only after the biological confirmation of low $\mathrm{Na}$ levels and the exclusions of other organic neurologic diseases.

Table I. Anamnesis and physical examination in hyponatremic patients.

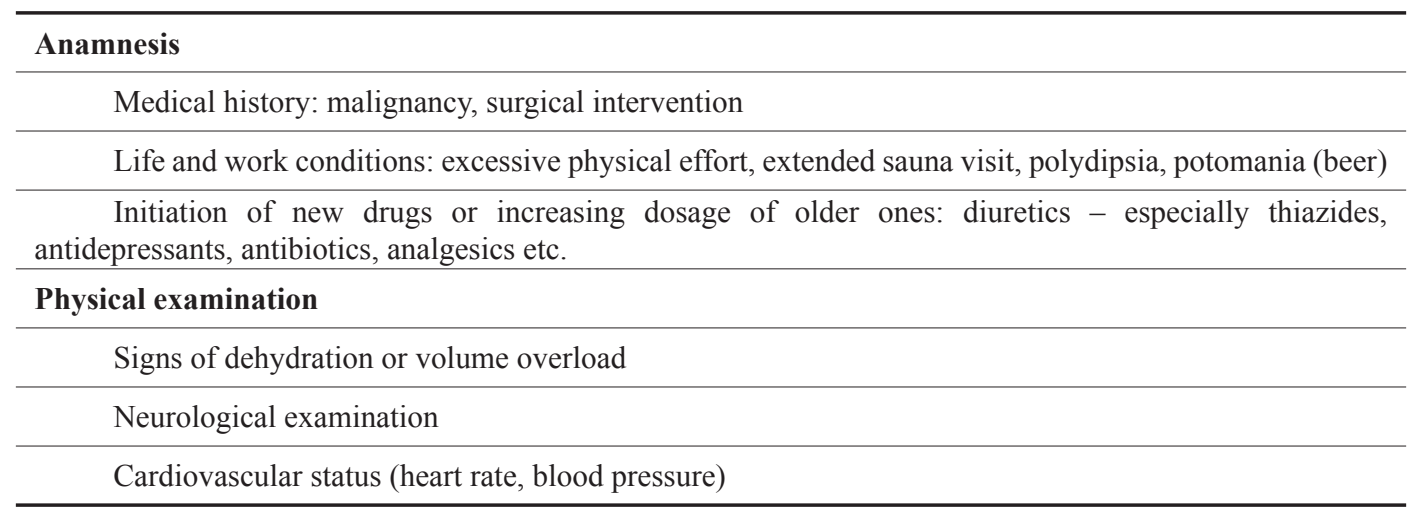

\section{Etiological diagnosis algorithm of hyponatremia}

Experts from different specialties often propose different diagnostic algorithms to facilitate the management of hyponatremic patients in the hospital settings. Traditionally, the diagnosis and treatment of hyponatremia have fallen within the remit of practitioners of nephrology and endocrinology. Therefore, to obtain a common and holistic view, the European Society of Intensive Care Medicine, the European Society of Endocrinology and the European Renal Association - European Dialysis and Transplant Association, represented by European Renal Best Practice, have developed the European Clinical Practice Guideline (2014) on the diagnostic approach and treatment of hyponatremia [6].

For the USA, guidelines on hyponatremia have been issued by an expert panel around Verbalis et al. in 2007, updated in 2013 [7]. The expert panel stressed as notable developments the importance of treating mild to moderate hyponatremia, and the establishment of efficacy and safety of vasopressin receptor antagonist therapy in more severe cases.

Most of the recommendations in relation to diagnosis, investigation and management in the emergency setting are convergent. There is variation in relation to the use of interventions after fluid restriction. Where there is a significant divergence, this is acknowledged in the text.

Using this guidelines and the facts known from previous studies, especially those extracted from critical care experience, where patients with severe hyponatremia are evaluated and treated, a diagnosis algorithm has been designed (Figure 1). This algorithm is useful both in acute and chronic conditions. It divides hyponatremia in three categories according to the plasmatic osmolality. Hypertonic hyponatremia (also pseudohyponatremia) and normotonic hyponatremia are two conditions that should be ruled out before managing hyponatremia. 


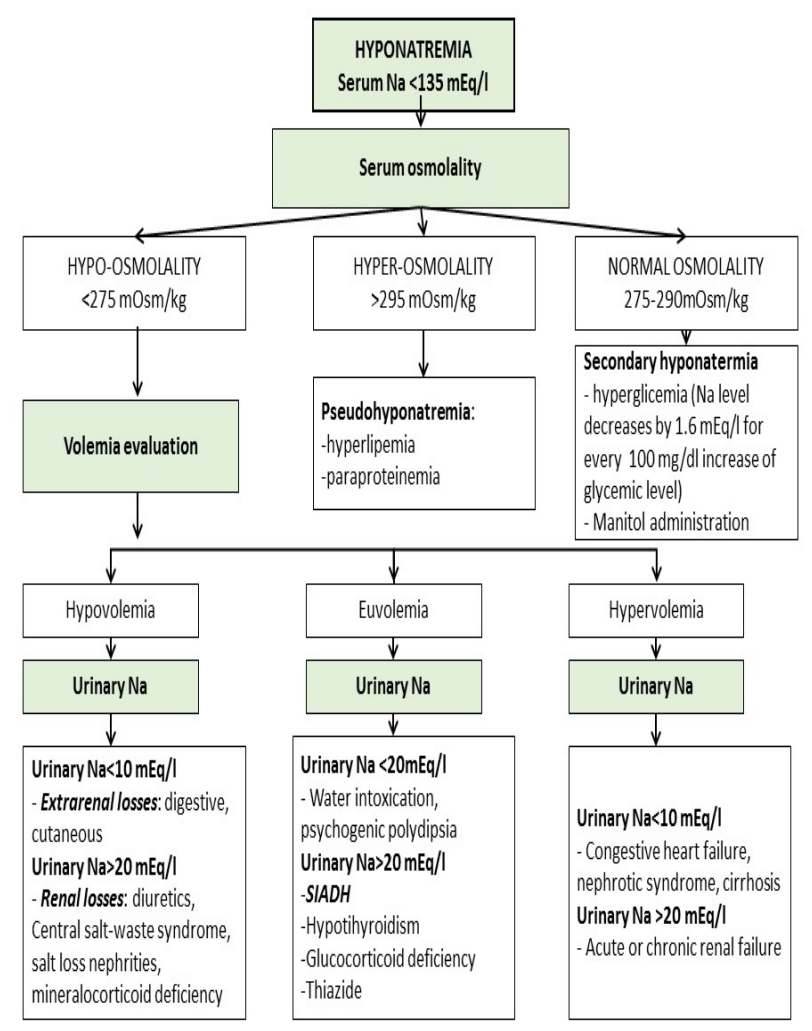

Figure 1. Etiological diagnosis algorithm of hyponatremia. Adapted from Schrier et al. [8] and Katzel et al. [9]. SIADH: Syndrome of Inappropriate Antidiuretic Hormone Secretion.

Pseudohyponatremia is due to marked elevation of lipids or proteins in plasma causing artifactual decrease in serum sodium concentration as a larger relative proportion of plasma is occupied by excess lipid or proteins. Hyponatremia with normal osmolality appears from osmotic shift of water from intracellular fluid to extracellular fluid due to additional solutes in plasma, e.g. glucose, mannitol, and radiographic contrast agents [8].

Once these two conditions are ruled out, the diagnosis of hypotonic hyponatremia depends on volemic status that could be appreciated clinically or by central venous pressure determination. In hypovolemic hyponatremia, there is a deficit of both total body water and sodium, but relatively less deficit of water. A history of digestive losses (vomiting, diarrhea), or renal losses (diuretic use, or hyperglycemia with glucosuria) along with increased clinical signs of dehydration (thirst, weight loss, orthostatic hypotension and tachycardia, and dry mucous membranes) are arguments for hypovolemic hyponatremia.

If the fluid and sodium losses are extra-renal, such as gastrointestinal losses, urinary $\mathrm{Na}$ should be less 10 $\mathrm{mEq} / \mathrm{l}$. On the contrary, if the loses of water and $\mathrm{Na}$ are of renal causes than urinary $\mathrm{Na}$ should be over $20 \mathrm{mEq} / \mathrm{l}$. Hypervolemic hyponatremia is related to systemic diseases causing water retention: congestive heart failure, nephrotic syndrome, cirrhosis or renal failure (acute or chronic). In this case the treatment is addressed to the underlying disease. These conditions are not difficult to identify if they are systematically searched [6].

Euvolemic hyponatremia is the most problematic from the etiological point of view. If the urinary $\mathrm{Na}$ excretion is below $20 \mathrm{mEq} / \mathrm{l}$, water intoxication or psychiatric disorders such as psychogenic polydipsia or potomania may be suspected. If the urinary $\mathrm{Na}$ excretion is over $20 \mathrm{mEq} / \mathrm{l}$ and hypothyroidism, glucocorticoid deficiency or thiazides were excluded, then the Syndrome of Inappropriate Antidiuretic Hormone Secretion (SIADH) may be the cause of hyponatremia. Clearly, this is an exclusion diagnosis with its own specific pathophysiologic mechanism, etiology and treatment.

\section{Definitions, etiology, pathophysiologic mechanisms, and diagnosis criteria of SIADH}

SIADH was first described by Schwartz and colleagues in 2 patients with bronchogenic lung carcinoma as early as 1957 [10].

SIADH is a disease categorized as hypotonic hyponatremia; it is considered euvolemic, even though a small amount of volume expansion is caused by excess of renal water reabsorption through inappropriate antidiuretic hormone $(\mathrm{ADH})$ secretion.

General anesthesia, nausea, pain, stress and a variety of drugs are non-specific but potent stimuli for the secretion of vasopressin and a frequent cause of SIADH in hospitalized 
patients. The most frequent causes of SIADH include cancers (e.g. small cell carcinoma of the lung) and diseases of the lung (e.g. pneumonia) or central nervous system (CNS) (e.g. subarachnoid hemorrhage) (Figure 2) [6,11].

Recently, several genetic disorders causing SIADH have been identified. The first is the polymorphisms in the genes encoding the hypothalamic osmoreceptor, transient receptor potential vanilloid type 4 (TRPV4), a gene that encodes for an osmosensitive calcium channel expressed in osmosensing neurons [12]. The second is a gain-offunction mutation in the vasopressin 2 receptor, resulting in a constitutively activated receptor causing increased water re-absorption and chronic hyponatremia [13].

The inappropriate secretion occurs independently from effective serum osmolality or circulating volume. It may result from increased pituitary secretion or from ectopic production. The excessive ADH secretion causes water retention by increasing water permeability in the renal collecting duct. Consequently, the increased glomerular filtration rate (GFR) due to the volume expansion and vasodilating effect of increased circulating atrial/brain natriuretic peptides can increase sodium excretion, but there is also decreased tubular transport of sodium due to unknown mechanism [11].

"The clinical description of the syndrome changed little since its original observation" [14] and the guidelines adopted a set of criteria for the diagnosis that are detailed in Table II.

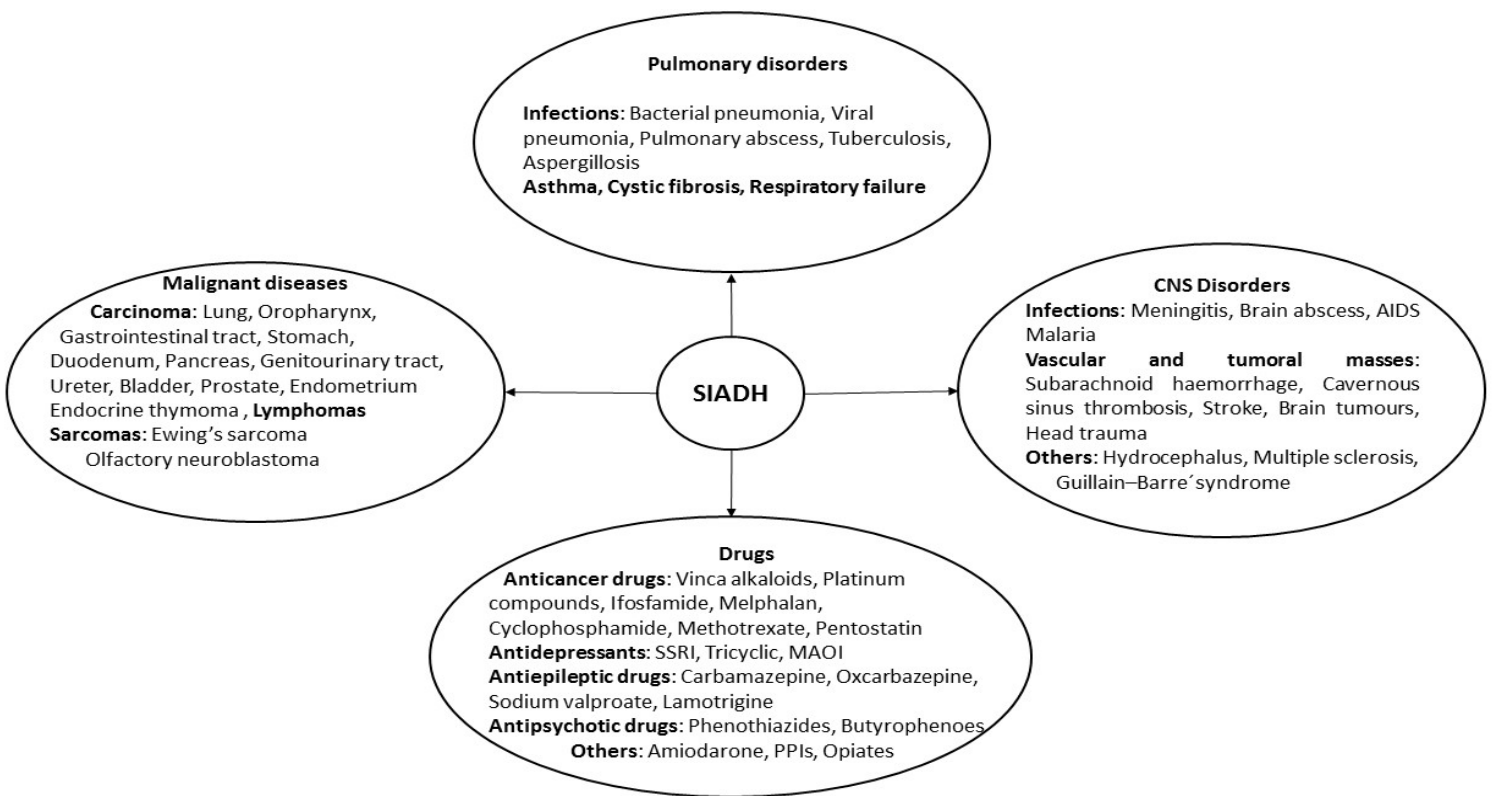

Figure 2. Causes of SIADH. Adapted from 2014 European Guideline [6] with modifications from Grant et al. [11]. CNS: central nervous system; SSRI: serotonin-specific reuptake inhibitors; MAOI: Monoamine oxidase inhibitors; PPIs: proton pomp inhibitors.

Table II. SIADH diagnosis criteria according to 2014 European Guideline [6].

\begin{tabular}{|l|}
\hline Essential criteria \\
\hline Effective serum osmolality $<275 \mathrm{mOsm} / \mathrm{kg}$ \\
\hline Urine osmolality $>100 \mathrm{mOsm} / \mathrm{kg}$ at some level of decreased effective osmolality \\
\hline Clinical euvolemia \\
\hline Urine sodium concentration $>30 \mathrm{mmol} / \mathrm{l}$ with normal dietary salt and water intake \\
\hline Absence of adrenal, thyroid, pituitary or renal insufficiency \\
\hline No recent use of diuretic agents \\
\hline Supplemental criteria \\
\hline Serum uric acid $<4 \mathrm{mg} / \mathrm{dl}$ \\
\hline Serum urea $<21.6 \mathrm{mg} / \mathrm{dl}$ \\
\hline Failure to correct hyponatremia after $0.9 \%$ saline infusion \\
\hline Fractional sodium excretion $>0.5 \%$ \\
\hline Fractional urea excretion $>55 \%$ \\
\hline Fractional uric acid excretion $>12 \%$ \\
\hline Correction of hyponatremia through fluid restriction \\
\hline
\end{tabular}




\section{Management of hyponatremia}

The correct treatment of hyponatremia in acute illness depends first and foremost on the correct diagnosis. Applying the above mentioned algorithm, only hypovolemic hyponatremia is appropriately treated by volume expansion with isotonic saline. Therefore, when hypovolemic hyponatremia is the suspected diagnosis, a trial of volume expansion with isotonic saline is certainly appropriate. If the diagnosis of hypovolemic hyponatremia is correct, the nonosmotic release of ADH should be suppressed, and the renal function improves with isotonic saline replacement. This corrects the hyponatremia over the next $24-48 \mathrm{~h}$. The efficiency of the treatment depends both on the reversibility of its cause and the type of its development (acute or chronic) [8]. Sometimes, in emergency it is difficult to determine whether the hyponatremia is acute or chronic. Thus, the severe symptomatic hyponatremia should be treated with saline infusion and the patients must be put under surveillance in order to establish the underlying cause and the onset type.

The European Guideline and the American Guideline have similar recommendations. They are well summed up in the latest article written by Aylwin et al. [15].

\section{Correction of acute hyponatremia}

Correction of acute severe and symptomatic hyponatremia with infusions of hypertonic saline (3\%): $100 \mathrm{~mL}$ over 10 minutes administered up to three times (or $150 \mathrm{ml}$ in 20 minutes administered 2 times), ideally in critical care unit. Acute hyponatremia with mild-tomoderate symptoms should be corrected with $3 \% \mathrm{NaCl}$ $(0.5-2 \mathrm{~mL} / \mathrm{kg} / \mathrm{h})$ infusions and the response should be monitored every 4-6 hours. Some authorities recognize the use of $1.8 \%$ hypertonic saline at $1 \mathrm{~mL} / \mathrm{kg} /$ hour as an alternative therapy in acute (and chronic) hyponatremia. The aim is to raise serum Na levels by $4-6 \mathrm{mEq} / 1$ in 6 hours in acute severe hyponatremia, followed by a pause for 12-24 hours. A small shift in serum sodium is sufficient to reduce intracranial pressure [15].

\section{Correction of chronic hyponatremia}

It is of great importance to stratify for the risk of osmotic demyelination syndrome (ODS): at-risk patients have serum $\mathrm{Na}<120 \mathrm{mEq} / 1$ for $>48$ hours, and high risk if serum $\mathrm{Na}<105 \mathrm{mEq} / \mathrm{l}$, or with hypokalemia, alcoholism, malnutrition, or advanced cirrhosis. The correction rate should not exceed 4-8 mEq/1 per day in low-risk patients with chronic SIADH and 4-6 mEq/l/day in patients at high risk of ODS. Na overcorrection should be avoided: $>8$ $\mathrm{mEq} / \mathrm{l} /$ day in high-risk patients, or $>12 \mathrm{mEq} / \mathrm{l} /$ day in lowrisk patients $[7,15]$. The maximal permitted correction is $18 \mathrm{mEq} / \mathrm{l}$ in 48 hours period. Sterns' Safety Rule of Sixes is an often cited and easily remembered rule emphasizing that symptom improvement can be achieved with a $\mathrm{Na}$ correction of $6 \mathrm{mEq} / \mathrm{l}$ ('six a day makes sense for safety; so six in six hours for severe Sx and stop') [16].

The clinician should monitor urine output during correction of hyponatremia as a high urine output may alert the clinician to overly rapid correction of serum $\mathrm{Na}$ and the serum Na each 4-6 hours with any active treatment until Na stabilizes.

The over-correction of hyponatremia is dangerous in patients with cortisol deficiency, or patients receiving desmopressin or thiazides without monitoring of $\mathrm{Na}$, urine osmolality and urine output. Life-threatening overcorrection can occur in 12 hours.

\section{Treatment of SIADH}

Severe or mild-to moderate hyponatremia in SIADH must be treated as any other acute hyponatremia with hypertonic saline infusion, respecting the recommendation described above.

The fluid restriction is recommended as first-line therapy in selected patients with chronic hyponatremia provided such patients do not meet the exclusions below. The typical fluid restriction should be $500 \mathrm{~mL} /$ day less than the 24 hour urine output (around $800 \mathrm{ml} /$ day fluid intake). The fluid restriction is forbidden if:

- the urine osmolality is $>500 \mathrm{mOsm} / \mathrm{kg}$

- the sum of urinary $\mathrm{Na}+$ urinary $\mathrm{K}>$ serum $\mathrm{Na}$ (Furst formula) [17].

- it leads to the discontinuation or delay of needed therapy (surgery, artificial nutrition, i.v. medication, chemotherapy etc.).

The fluid restriction alone should be discontinued if the initial $\mathrm{Na}$ correction is less than $2 \mathrm{mEq} / \mathrm{l}$ in the first 24-48 hours. If an effective fluid restriction fails to lead to correction after a few days the diagnosis of euvolemic hyponatremia should be reconsidered.

This form of treatment for hyponatremia is often slow and can be difficult for patients to maintain in the long term because of hidden liquids in foods and discomfort with thirst [18].

Medication. The 2014 European Guideline recommends against the use of vasopressin antagonists for the treatment of SIADH hyponatremia. The arguments are represented by a negative risk/benefit ratio with concern regarding the toxicity profiles of these drugs [19]. Neither demeclocycline and lithium, have been proven of any help, so the European Guideline advises against their use for management of any degree of chronic hyponatremia in patients with SIADH [6].

Over the ocean, the Guideline updated in 2013, recommend the lowest recommended standard dose of a vasopressin V2-receptor blocker in hyponatremic patients with suspected SIADH without a therapeutic effect of fluid-intake restriction [7]. Empiric V2-receptor antagonist represents a rescue therapy. Low-dose tolvaptan was shown to significantly improve hyponatremia (by $3-4 \mathrm{mmol} / \mathrm{l}$ ) within 4 days when compared to placebo [20]. Alternatively, conivaptan is approved for SIADH in the United States. Conivaptan selectively targets the V1a and V2 receptors and can be administered intravenously in patients who are 
unable to take drugs orally [21].

The UK adopts a similar position regarding the use of pharmacological therapy for SIADH: "DO consider pharmacological therapy for SIADH where hyponatremia persists and where fluid restriction is ineffective, impractical or unpalatable" [15], as the drugs obtained their license in the EU recently.

In essence, SIADH with acute hyponatremia should be treated with i.v. saline solutions. For chronic hyponatremia, fluid restriction is the first line therapy. Vaptans might be a rescue therapeutic tool for SIADH when applied in cautiously selected cases, under medical surveillance and for a limited period of time. To date, safety data on long-term use of V2-receptor blockers have not been published, especially not for the use of a combined $\mathrm{V} 1 \mathrm{a} / \mathrm{V} 2$ receptor blockade.

\section{Conclusions}

Hyponatremia is a common electrolyte disturbance which should not be ignored, even if asymptomatic. Once identified, the severity and the type of onset should be evaluated. Correct diagnosis of the etiology of hyponatremia is critical, both to determine correct management and prognosis. In practice, interpretation of clinical and biochemical findings is difficult due to a combination of the complex, multifactorial etiology encountered frequently. Thus, a clinically applicable diagnostic algorithm and the use of diagnosis criteria to define SIADH with accuracy is helpful to avoid misinterpretation. Acute hyponatremia should be promptly corrected with saline solutions. Chronic hyponatremia should be investigated to establish etiology.

Future work should focus on improving current diagnostic tools. New and emerging therapies should focus on gathering evidence of their safety and benefits in terms of clinical outcomes and cost effectiveness

\section{References}

1. Upadhyay A, Jaber BL, Madias NE. Incidence and prevalence of hyponatremia. Am J Med. 2006;119(7 Suppl 1):S30-S35.

2. Hoorn EJ, Lindemans J, Zietse R. Development of severe hyponatraemia in hospitalized patients: treatment-related risk factors and inadequate management. Nephrol Dial Transplant. 2006;21:70-76.

3. Mannesse CK, Vondeling AM, van Marum RJ, van Solinge WW, Egberts TC, Jansen PA. Prevalence of hyponatremia on geriatric wards compared to other settings over four decades: A systematic review. Ageing Res Rev. 2013;12(1):165-173.

4. Clayton JA, Le Jeune IR, Hall IP. Severe hyponatraemia in medical in-patients: aetiology, assessment and outcome. QJM. 2006;99(8):505-511.

5. Ball SG. How I approach hyponatraemia. Clin Med. 2013;13(3):291-295.
6. Spasovski G, Vanholder R, Allolio B, Annane D, Ball S, Bichet $\mathrm{D}$, et al. Clinical practice guideline on diagnosis and treatment of hyponatraemia. Eur J Endocrinol. 2014;170(3):G1-G47.

7. Verbalis JG, Goldsmith SR, Greenberg A, Korzelius C, Schrier RW, Sterns RH, et al. Diagnosis, evaluation, and treatment of hyponatremia: expert panel recommendations. Am J Med. 2013;126(10 Suppl 1):S1-S42.

8. Schrier RW, Bansal S. Diagnosis and management of hyponatremia in acute illness. Curr Opin Crit Care. 2008;14(6):627-634.

9. Katzel J. Fluide, electroliti, acizi, baze. Ghidul medicului de garda [Fluids, electrolytes, acids, bases. A guide for the physician on duty]. Targu-Mures: Farmamedia; 2011. p. 32-33.

10. Schwartz WB, Bennett W, Curlop S Bartter FC. A syndrome of renal sodium loss and hyponatremia probably resulting from inappropriate secretion of antidiuretic hormone. Am J Med. 1957;23:529-542.

11. Grant P, Ayuk J, Bouloux PM, Cohen M, Cranston I, Murray RD, et al. The diagnosis and management of inpatient hyponatraemia and SIADH. Eur J Clin Invest. 2015;45(8):888894.

12. Tian W, Fu Y, Garcia-Elias A, Fernández-Fernández JM, Vicente R, Kramer PL, et al. A loss-of-function nonsynonymous polymorphism in the osmoregulatory TRPV4 gene is associated with human hyponatremia. Proc Natl Acad Sci U S A. 2009;106(33):14034-14039.

13. Gitelman SE, Feldman BJ, Rosenthal SM. Nephrogenic syndrome of inappropriate antidiuresis: a novel disorder in water balance in pediatric patients. Am J Med. 2006;119(7 Suppl 1):S54-S58.

14. Grohé C, Berardi R, Burst V. Hyponatraemia - SIADH in lung cancer diagnostic and treatment algorithms. Crit Rev Oncol Hematol. 2015;96(1):1-8.

15. Aylwin S, Burst V, Peri A, Runkle I, Thatcher N. " Dos and don'ts" in the management of hyponatremia. Curr Med Res Opin. 2015;31(9):1755-1761

16. Sterns R, Hix JK, Silver S. Treating profound hyponatremia: a strategy for controlled correction. Am J Kid Dis. 2010;56:774779 .

17. Decaux G, Musch W. Clinical laboratory evaluation of the syndrome of inappropriate secretion of antidiuretic hormone. Clin J Am Soc Nephrol. 2008;3(4):1175-1184.

18. Soiza RL, Talbot HSC. Management of hyponatraemia in older people: old threats and new opportunities. Ther Adv Drug Saf. 2011;2(1):9-17.

19. Torres VE, Chapman AB, Devuyst O, Gansevoort RT, Grantham JJ, Higashihara E, et al. Tolvaptan in patients with autosomal dominant polycystic kidney disease. N Engl J Med. 2012;367(25):2407-2418.

20. Verbalis JG, Adler S, Schrier RW, Berl T, Zhao Q, Czerwiec FS, et al. Efficacy and safety of oral tolvaptan therapy in patients with the syndrome of inappropriate antidiuretic hormone secretion. Eur J Endocrinol. 2011;164(5):725-732.

21. Li-Ng M, Verbalis JG. Conivaptan: Evidence supporting its therapeutic use in hyponatremia. Core Evid. 2010;4:83-92. 